\title{
Metodología para el cálculo del desempeño térmico de turbuladores helicoidales en calderas de vapor: modelo matemático y simulación
}

\author{
Methodology for calculating the thermal performance of helical turbulators in \\ steam boilers: mathematical model and simulation
}

\author{
C. R. Rubio-Ramirez iD, G. Martheyn-Lizarazo iD, L. E. Vera- Duarte iD
}

\begin{abstract}
Resumen- El desempeño térmico que hoy en dia ofrecen las maquinas generadoras de vapor es medido principalmente por la cantidad de calor que es aprovechado. Para incrementar la transferencia de calor dentro los recipientes de presión se usan turbuladores que distribuyen uniformemente el contenido de gases de combustión al paso del haz de tubos. En este artículo se analizó el desempeño de los turbuladores helicoidales al interior de una sección del haz de tubos, presente en el segundo paso de calentamiento de una caldera pirotubular. Se deduce el modelo matemático de la transferencia de calor de los gases de combustión de acuerdo al régimen de fluidez. Para la caracterización del modelo se obtienen los contornos de imagen a partir de fotogrametría digital, apoyados en el software CAD para el procesamiento del sólido en 3D. Se realiza la simulación de los flujos de gases que atraviesan estos dispositivos, calculando la temperatura de los gases, componentes de velocidad, transferencia de calor y caídas de presión, comparando los resultados con un entorno sin turbulador aumentando la transferencia de energía en un 31,23\%. Así mismo se establece un patrón de la trayectoria que obtienen los gases en presencia de los turbuladores.
\end{abstract}

Palabras clave - Caldera de vapor, modelo matemático, simulación, turbulador helicoidal.

Abstract - Today, the thermal performance offered by steam generators is measured mainly by the amount of heat that is used. To increase the heat transfer within the pressure vessels, turbulators are used for distributing the gases fluxes passaging through the tube bundle. This article analyzed the performance of helical turbulators inside a section of tube bundle, located in the second heating step of a pirotubular boiler. The mathematical model of the heat transfer of gases fluxes is deduced according to

Este manuscrito fue enviado el 15 de enero de 2019 y aceptado el 23 de septiembre de 2019. Este estudio hace parte del proyecto de investigación en máquinas de vapor, del Grupo de Investigación de Fluidos y Plantas Térmicas FLUTER financiado por la Universidad Francisco de Paula Santander, Cúcuta. Vigencia 2020.

C. Rubio-Ramirez, Escola de Engenharia, Universidade Federal do Rio Grande do Sul, Porto Alegre, Brasil. (cristian.ricardo@ufrgs.br)

${ }^{2}$ G. Martheyn-Lizarazo, Facultad de Ingenieria, Universidad Francisco de Paula Santander, Cúcuta, Colombia. (guillermoalfredomli@ufps.edu.co)

${ }^{3}$ L. E. Vera C. R. Rubio-Ramirez , G. Martheyn-Lizarazo , L. E. VeraDuarte. Facultad de Ingenieria, Universidad Francisco de Paula Santander, Cúcuta, Colombia. (luisemiliovd@ufps.edu.co). the flow rate. For image characterization, contours are obtained from digital photogrammetry and the 3D solid processing was supported by software CAD. The simulation of flows gases that pass through these devices is performed, calculating the temperature of gases, speed components, heat transfer and pressure drops, comparing the results with an environment without turbulator increasing the energy transfer by $31,23 \%$. A pattern of the trajectory that gases obtain in the presence of turbulators is also established.

Index Terms - Steam boiler, mathematical model, simulation, helical turbulator.

\section{INTRODUCCION}

$\mathrm{L}$ AS calderas de vapor son fundamentales en la industria ya que cumplen la función de agregarle energía a un fluido como lo es el agua, que luego se utiliza para múltiples aplicaciones entre los cuales están la generación de energía eléctrica por medio de turbinas, climatización de espacios y en la desinfección de alimentos, entre otros.

El desempeño de los dispositivos de alta presión es evaluado por su eficiencia. La eficiencia de una caldera, corresponde a la razón entre el calor absorbido por el agua y el calor liberado en el equipo por la combustión. Es por eso, que las investigaciones relacionadas con calderas buscan aumentar la eficiencia mediante el desarrollo de diferentes mecanismos que mejoren la transmisión de calor ya sea por áreas de contacto, espesores de materiales y aislantes o dispositivos anexos como turbuladores o aletas.

Los turbuladores son elementos adicionales que se introducen en los intercambiadores de calor, especialmente en los haces de tubos de las calderas con varios pasos. Estos se instalan con el fin de distribuir el flujo de gases de combustión generalmente en régimen laminar o en transición por el borde y así generar calor extra por fricción con la pared [1]. La presencia de estos dispositivos mejora la velocidad de transferencia de calor y vuelve homogéneo el calentamiento de la caldera, reduciendo los consumos y por consiguiente las emisiones de gases contaminantes a la atmósfera.

Existen diferentes tipos de turbuladores helicoidales, según Hartnett e Irvine [2], entre los cuales se encuentran: 
- Turbuladores de longitud completa: ocupan el largo completo del tubo del intercambiador. Los más comunes son de tipo resorte hechos de acero ordinario

- Turbuladores modificados: que incluyen un tramo corto de elemento colocado en la entrada del tubo, y presenta variaciones marcadas en la geometría del turbulador. Por lo general están hechos en fundición

- Turbuladores compuestos: cuando se agregan más de un dispositivo para mejorar el comportamiento de turbulencia. Los más usados son de tipo espiral hechos en hierro, acero normal o acero inoxidable.

Los turbuladores de longitud completa y compuestos son fabricados en múltiples longitudes por lo que se adaptan a cada espacio de trabajo. Al momento de seleccionarlos se recomienda tener una holgura de unos milímetros con las paredes de la caldera, ya que tienden a dilatarse por las altas temperaturas. Por otro lado, los turbuladores de fundición no presentan este problema, y por eso están hechos específicamente para cada generador de vapor.

Debido a lo compleja que resulta la geometría de los turbuladores modificados, las investigaciones que tienen como punto focal estos elementos son muy pocas, por ello se parte del concepto de ingeniería inversa para realizar adecuadamente los cálculos de transferencia de calor y velocidad de fluidos.

Para este estudio se toma como prototipo una caldera de tubos de fuego con una potencia nominal de $10 \mathrm{BHP}$, la cual cuenta con turbuladores hechos en fundición gris, es decir, de tipo modificados. En base a esto, se propone un modelo matemático según el régimen de velocidad de los fluidos y se resuelve apoyados en el software EES. Aplicando los conceptos de ingeniería inversa, se emplea el método de fotogrametría digital, para obtener de forma detallada la geometría de interés. Anexo, la simulación se logra por medio del entorno de Flow Simulation, el cual se basa en la teoría de turbulencia K-épsilon.

\section{Metodologia}

\section{A. Modelo matemático}

El modelo matemático parte de la explicación de un fenómeno físico complejo resuelto por medio de relaciones entre variables de operación y parámetros de funcionamiento. A continuación, en la Tabla 1, se muestra la nomenclatura más relevante del modelo matemático, donde se detallan variables intrínsecas y extrínsecas del proceso, así como sus respectivas unidades.

Para el cálculo del coeficiente de transferencia de calor por convección se debe establecer el régimen en que circulan los gases dentro del tubo, para lo cual se calcula el número de Reynolds, basados en la ecuación que define el flujo de gases dentro de un tubo circular de sección uniforme [3].

$$
\operatorname{Re}=\frac{4 * \dot{m}_{\text {gases }}}{\pi * \mathrm{D}_{\text {int }} * \mu_{\text {gases }}}
$$

El número de Reynolds evalúa como es el flujo, es decir, indica que tan desordenadas viajan las partículas en movimiento. En la Tabla II, se identifica en que régimen se encuentra el flujo de gases de acuerdo al número de Reynolds [4].
TABLA I

NOMENCLATURA CARACTERÍSTICA DEL MODELO MATEMÁTICO

\begin{tabular}{|c|c|c|}
\hline Símbolo & Significado & Unidades \\
\hline $\operatorname{Re}$ & Numero de Reynolds & \\
\hline$\dot{\mathrm{m}}_{\text {gases }}$ & Flujo másico de gases & $\mathrm{kg} / \mathrm{s}$ \\
\hline $\mathrm{D}_{\text {int }}$ & Diámetro interno del tubo & $\mathrm{m}$ \\
\hline$\mu_{\text {gases }}$ & Viscosidad dinámica de los gases & $\mathrm{kg} / \mathrm{m} \cdot \mathrm{s}$ \\
\hline $\mathrm{Nu}_{\mathrm{D}}$ & Numero de Nusselt sin Turbulador & \\
\hline $\mathrm{Nu}_{\mathrm{T}}$ & Numero de Nusselt con turbulador & \\
\hline $\mathrm{fr}$ & Factor de Fricción & \\
\hline $\operatorname{Pr}$ & Numero de Prandtl & \\
\hline HL & Longitud de hélice del turbulador & $\mathrm{m}$ \\
\hline $\mathrm{d}$ & Diámetro del turbulador & $\mathrm{m}$ \\
\hline$\varpi$ & Espesor de la hélice del turbulador & $\mathrm{m}$ \\
\hline $\mathrm{Tm}$ & Temperatura media de los gases & K \\
\hline $\mathrm{T} 2_{\text {gases }}$ & $\begin{array}{l}\text { Temperatura de los gases a la } \\
\text { entrada del tubo }\end{array}$ & $\mathrm{K}$ \\
\hline $\mathrm{T} 3_{\text {gases }}$ & $\begin{array}{l}\text { Temperatura de los gases a la } \\
\text { salida del tubo }\end{array}$ & $\mathrm{K}$ \\
\hline $\mathrm{T}_{\mathrm{S}}$ & $\begin{array}{l}\text { Temperatura superficial interna } \\
\text { del tubo }\end{array}$ & $\mathrm{K}$ \\
\hline $\mathrm{K}_{\text {gases }}$ & $\begin{array}{l}\text { Conductividad térmica de los } \\
\text { gases }\end{array}$ & $\mathrm{W} / \mathrm{m} \cdot \mathrm{K}$ \\
\hline$Q_{\text {conv }}$ & Calor de convección & W \\
\hline $\mathrm{h}_{\text {conv }}$ & $\begin{array}{l}\text { Coeficiente de transferencia de } \\
\text { calor por convección }\end{array}$ & $\mathrm{W} / \mathrm{m}^{2} \cdot \mathrm{K}$ \\
\hline$\Delta \mathrm{P}$ & Caída de presión & $\mathrm{kPa}$ \\
\hline$\rho_{\text {gases }}$ & Densidad de los gases & $\mathrm{kg} / \mathrm{m}^{3}$ \\
\hline$V_{\text {gases }}$ & Velocidad de los gases & $\mathrm{m} / \mathrm{s}$ \\
\hline$\phi$ & Angulo de hélice del turbulador & \\
\hline
\end{tabular}

TABLA II

DESCRIPCIÓN DEL RÉGIMEN DE FLUJO

Régimen del fluido

$\begin{array}{lc}\text { Flujo laminar } & R e \leq 2300 \\ \text { Flujo en transición } & 2300<R e<10000 \\ \text { Flujo turbulento } & R e \geq 10000\end{array}$

De acuerdo al régimen en el que se encuentran los gases circulando dentro del tubo, se selecciona la correlación apropiada para el número de Nusselt $\left(N u_{D}\right)$ de entre las siguientes ecuaciones [5].

Para flujo laminar completamente desarrollado, y temperatura de la pared constante

$$
\mathrm{Nu}_{\mathrm{D}}=3,66
$$

Para flujo en transición y número de Prandtl $0,5<\operatorname{Pr}<$ 2000

$$
\mathrm{Nu}_{\mathrm{D}}=\frac{\left(\frac{\mathrm{fr}}{2}\right) *(\mathrm{Re}-1000) * \mathrm{Pr}}{1+12,7 *\left(\frac{\mathrm{fr}}{2}\right)^{1 / 2} *\left(\mathrm{Pr}^{2} / 3-1\right)}
$$

Donde el factor de fricción $(f r)$ es

$$
\text { fr }=(1,58 \ln * \operatorname{Re}-3,28)^{-2}
$$

Al momento que los gases entran al haz de tubos, estos ya han perdido determinada cantidad de energía por dos motivos: primero, se trasfirió calor por parte de los gases de combustión hacia el agua en la sección del hogar; segundo, los gases sufren contracciones y expansiones abruptas lo que generan altas caídas de presión afectando directamente la velocidad de 
los fluidos [6]. Por esto se considera que los flujos solo estarán en flujo laminar o en transición.

En la Fig. 1, se muestra la geometría del turbulador presente en la caldera de estudio, señalando los parámetros de relevancia.

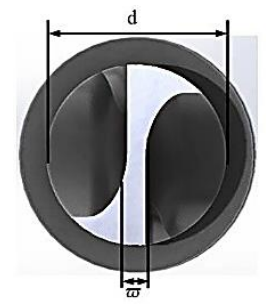

(a)

(b)

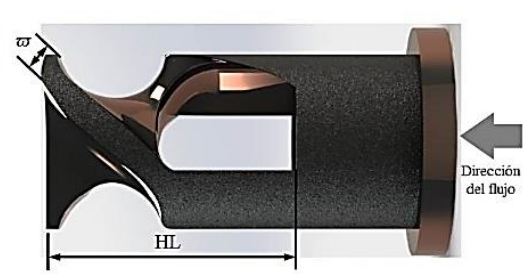

Fig. 1. Parámetros de medición en la geometría de un turbulador. (a) vista frontal (b) vista lateral. Fuente. Elaboración propia.

Las aplicaciones de los procesos térmicos generalmente se relacionan con la producción de vapor por calentamiento, fluidos en estados turbulentos o flujos laminares en presencia de turbuladores. Para analizar este tipo de fenómenos, se selecciona el parámetro de Temperatura de Pared Uniforme (Uniform Wall Temperature, UWT). Por eso la ecuación que describe el Nusselt del turbulador $\left(N u_{T}\right)$ es descrita por [7] y [8].

$$
\mathrm{Nu}_{\mathrm{T}}=4,612 * \theta\left\{\begin{array}{c}
{\left[\begin{array}{c}
\left(1+0,0951 \mathrm{Gz}^{0,894}\right)^{2,5} \\
+6,413 * 10^{-9} \\
*\left(\mathrm{Sw} * \mathrm{Pr}^{0,391}\right)^{3,853}
\end{array}\right]^{0,2}} \\
+2,132 * 10^{-14}\left(\mathrm{Re}_{\mathrm{ax}} * \mathrm{Ra}\right)^{2,23}
\end{array}\right\}^{0,1}
$$

Se describen cada uno de los parámetros que influyen en la ecuación de Nusselt a través de un flujo en régimen laminar.

Relación de giro del turbulador

$$
\mathrm{Y}=\frac{\mathrm{HL}}{\mathrm{d}}
$$

Parámetro adimensional de giro

$$
\mathrm{SW}=\frac{\mathrm{Re}_{\mathrm{S}}}{\sqrt{\mathrm{Y}}}
$$

Numero de Reynolds basado en la velocidad de giro

$$
\operatorname{Re}_{\mathrm{s}}=\frac{\rho * \mathrm{~V}_{\mathrm{s}} * \mathrm{~d}}{\mu}
$$

Velocidad de giro en el turbulador

$$
\mathrm{V}_{\mathrm{S}}=\mathrm{V}_{\mathrm{a}}\left[1+\left(\frac{\pi}{2 \mathrm{Y}}\right)^{2}\right]^{1 / 2}
$$

Velocidad de flujo axial

$$
\mathrm{V}_{\mathrm{a}}=\frac{\dot{\mathrm{m}}}{\rho * \mathrm{~A}_{\mathrm{c}}}
$$

Sección transversal del flujo libre sobre el turbulador

$$
\mathrm{A}_{\mathrm{c}}=\left(\frac{\pi * \mathrm{~d}^{2}}{4}\right)-\varpi \mathrm{d}
$$

Número de Reynolds característico basado en velocidad axial

$$
\operatorname{Re}_{\mathrm{ax}}=\frac{\dot{\mathrm{m}} * \mu}{\left(\frac{\pi * \mathrm{~d}}{4}\right)-\varpi \mathrm{d}}
$$

Número de Graetz

$$
\mathbf{G z}=\frac{\dot{\mathbf{m}} * \mathbf{C p}}{\mathbf{k} * \mathbf{L}}
$$

Número de Rayleigh

$$
\mathbf{R a}=\mathbf{G r} * \mathbf{P r}
$$

Número de Grashof

$$
\mathbf{G r}=\frac{\mathbf{g} * \mathbf{\rho}^{2} * \mathbf{d}^{3} * \boldsymbol{\beta} * \mathrm{Ts}}{\boldsymbol{\mu}^{2}}
$$

Relación de viscosidades

$$
\theta=\left(\frac{\mu_{\mathrm{m}}}{\mu_{\mathrm{s}}}\right)^{0,14}
$$

Con el número de Nusselt calculado se obtiene el coeficiente de convección interno mediante la relación [9]

$$
\mathrm{h}_{\text {conv }}=\frac{\mathrm{Nu}_{\mathrm{T} \sim \mathrm{D} * \mathrm{~K}_{\text {gases }}}}{\mathrm{D}_{\text {int }}}
$$

También se calcula el calor de convección generado por el turbulador, basado en la ley de enfriamiento de Newton:

$$
\mathrm{Q}_{\text {conv }}=\mathrm{A}_{\text {int }} * \mathrm{~h}_{\text {conv }} *\left(\mathrm{~T}_{\mathrm{m}}-\mathrm{T}_{\mathrm{s}}\right)
$$

Donde

$$
\mathrm{Tm}=\frac{\left(\mathrm{T} 2_{\text {gases }}+\mathrm{T} 3_{\text {gases }}\right)}{2}
$$

Se obtiene la caída de presión donde el factor de fricción fr depende principalmente del número de Reynolds [4].

$$
\Delta \mathrm{P}=\mathrm{fr} *\left(\frac{\rho_{\text {gases }} * \mathrm{~V}_{\text {gases }}}{2 * \mathrm{D}_{\text {int }}}\right)
$$

Para el flujo laminar completamente desarrollado la ecuación que define el parámetro de fricción está dado por la ecuación de fricción de Darcy

$$
\mathrm{fr}=\frac{64}{\mathrm{Re}}
$$

En el caso del flujo en transición se usa el factor de fricción propuesto en (4).

Para calcular el factor de fricción " $f r$ " en un turbulador se debe tener en cuenta la relación entre el número de Reynolds y el parámetro de geometría helicoidal.

En las ecuaciones 22 y 23 se observan los rangos de selección dependiendo del caso de estudio.

Estas ecuaciones fueron propuestas por Date y Singham [10] y retomadas por Manglik \& Bergles [8]:

$$
\mathrm{fr}=\left(8,8201-2,1193 \mathrm{Y}+0,2108 \mathrm{Y}^{2}-0,0069 \mathrm{Y}^{3}\right)\left(\frac{\mathrm{Re}}{\mathrm{Y}}\right)^{-0,7}
$$

Si cumple con el requisito de $\frac{R e}{Y}>100$

Para el caso contrario, es decir si $\frac{R e}{Y}<100$, se emplea esta ecuación.

$$
\mathrm{fr}=38,4 *\left(\frac{\mathrm{Re}}{\mathrm{Y}}\right)^{-0,95}
$$


Para calcular la caída de presión en presencia de un turbulador se emplea la velocidad axial de giro, descrita en (10).

\section{B. Fotogrametría digital}

Cuando se hace una documentación 3D por medio de fotogrametría digital, lo recomendado es contar con un guion de trabajo y con equipos especializados como cámaras métricas, lentes específicos para las mismas, set de luces para contrarrestar posibles sombras muy marcadas, entre otros, [11].

En el presente trabajo se utiliza la metodología basada en el análisis multifocal de la imagen, lo que permite verificar el posicionamiento correcto de los puntos guías comparando las imágenes en un rango sucesivo de movimiento. Esta técnica hace parte de la fotogrametría de objeto cercano recomendable para objetos de menor escala [12].

Para el caso de estudio se optó por un espacio cerrado, con luces artificiales tipo halógenas. El objeto se ubicó sobre un entorno blanco (mesa y fondo). La cámara permaneció estática sobre su eje trípode, y se roto el objeto de estudio cada $5^{\circ}$. Debido a la simetría del objeto se tomaron fotografías para la pieza rotada $180^{\circ}$, también se toma foto de la vista de techo, perpendicular al inicio y al final de la base.

Se considera de sumo cuidado definir bien los contornos de la curva, ya que es ella quien controla en gran parte el comportamiento del fluido cuando pasa a través del turbulador.

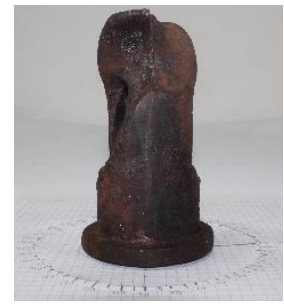

(a)

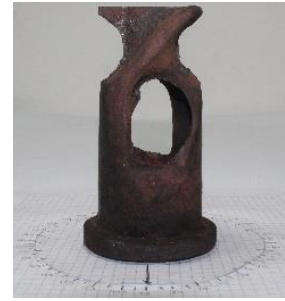

(b)

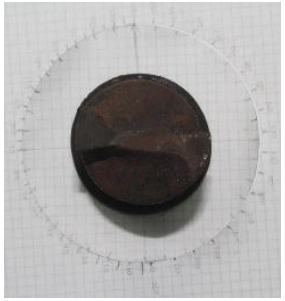

(c)
Fig. 2. Principales vistas del objeto de estudio. (a) vista a $0^{\circ}$, (b) vista a $90^{\circ}$, (c) vista de techo. Fuente. Elaboración propia.

Para la generación del sólido en 3D, se crearon planos alrededor de un eje de simetría y como base se generó un cilindro, de acuerdo a las medidas del objeto. Para la reproducción de la curva de turbulencia se realiza la toma de puntos a través de la creación de planos. Se coloca la respectiva imagen de cada grado, tomando cero grados como el inicio del área compleja. Los puntos describen la trayectoria de la curva la cual pasa de ser un bosquejo en 2D a un objeto macizo por recubrimientos y extrusiones. Por último, se añaden los detalles de inclinaciones, espesores y contornos, para lograr un modelo geométrico acorde a la realidad.

Para agregar una mejor apariencia y poder apreciar la textura del material original, se realiza el proceso de renderizado, basados en el cálculo de iluminación global o indirecta. Esto permitirá que la simulación de condiciones físicas sea lo suficientemente realista. [13]

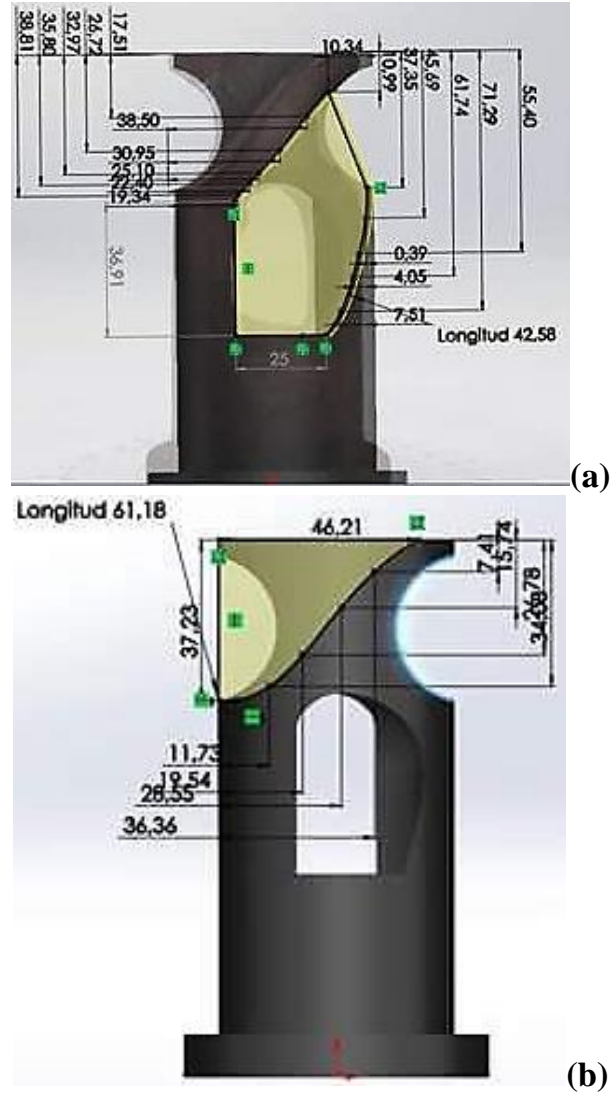

Fig. 3. Construcción del modelo 3D del turbulador (a) vista de la fotografía $90^{\circ}$ en su plano de trabajo, (b) corrección de detalles. Fuente. Elaboración propia.

\section{Simulación computacional}

Una vez obtenido el modelo 3D del objeto de estudio, este se carga al módulo Flow Simulation, en el cual se establecen los parámetros de operación de la caldera, como composición química de los gases de combustión, sus propiedades termofísicas como densidad, viscosidad dinámica, conductividad térmica y poder calorífico, evaluados a la temperatura media (diferencia entre la temperatura de entrada y de salida), además de flujo másico y presión de operación.

Flow simulation tiene la función de pared modificada con la cual admite el modelo de transición laminar-turbulento, además representa la compresibilidad del flujo automáticamente. El módulo de simulación se basa en el modelo de turbulencia $\mathrm{k}-\varepsilon$, el cual describe los flujos laminares, turbulentos y transicionales instintivamente [14].

Como fase inicial se delimita el volumen de control, y se inicia el módulo de ayudante de simulación "Wizard", seleccionando el sistema de unidades, y los mecanismos de transferencia de calor. Adicional se agrega el eje de referencia con respecto al objeto de estudio, y se definen los gases que intervienen en la simulación.

Para la simulación se define el material del sólido, ya que influye en la conducción de calor. Además, se ingresan las condiciones iniciales, como temperatura de los muros del tubo $\mathrm{y}$ temperatura del ambiente [15]. 


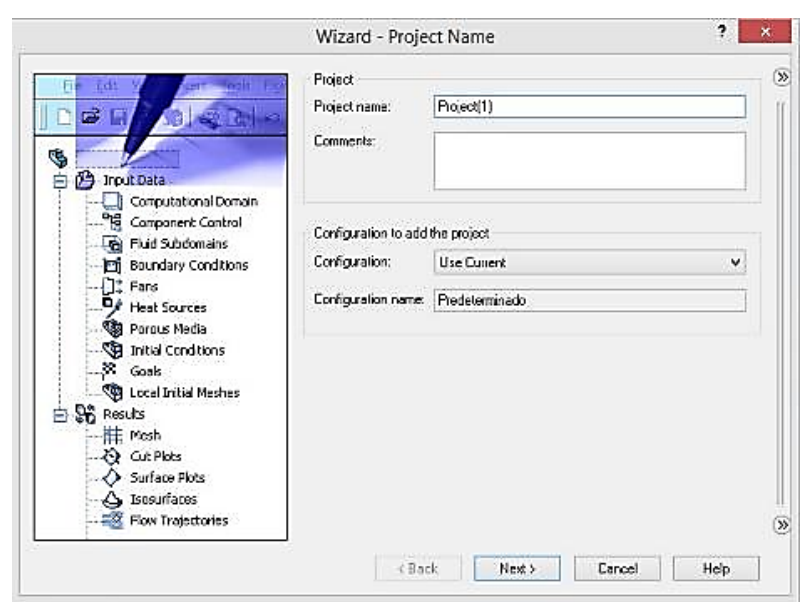

Fig. 4. Cuadro de dialogo asistente de simulación. Fuente. Solidworks 2015.

En el cuadro de dialogo Property Manager, se limitan las condiciones de frontera, definiendo los contornos que albergan los gases con sus respectivas fracciones másicas, detallando también un "inlet" o entrada del fluido, un "outlet" o salida a la atmosfera y un "outer wall" o condición de muro externo. Como paso final se crean los "goals" o metas, que son las propiedades que se quieren medir durante el proceso de simulación.

\section{RESUltados}

Los resultados se obtuvieron para la geometría una caldera de potencia nominal de 10 BHP tipo pirotubular, Fig. 5. Además, se tomaron en cuenta sus variables en la combustión, temperatura de llama y constantes termofísicas de los materiales y fluidos involucrados.

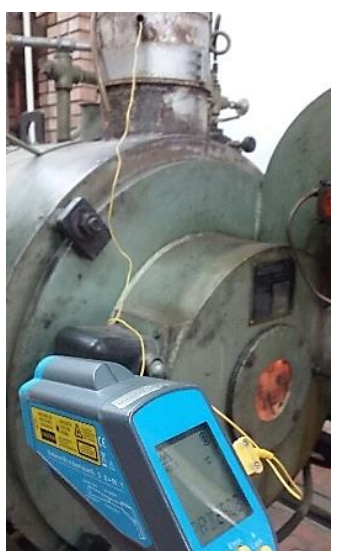

Fig. 5. Toma de datos experimentales, caldera 10 BHP.

Fuente. Elaboración propia.

Las medidas utilizadas en los cálculos están dadas en sistema internacional, sin embargo, algunas medidas de presión están dadas en el sistema inglés, ya que es el característico de la caldera de estudio, como se muestra a continuación
TABLA III

DATOS DE OPERACIÓN DE LA CALDERA

\begin{tabular}{ll}
\hline \hline \multicolumn{1}{c}{ Descripción } & \multicolumn{1}{c}{ Dato } \\
\hline Capacidad nominal & $10 \mathrm{BHP}$ \\
Tipo de combustible & $\mathrm{ACPM}$ \\
Consumo de combustible nominal & $3 \mathrm{gal} / \mathrm{h}$ \\
Consumo de combustible real & $1,2 \mathrm{gal} / \mathrm{h}$ \\
Exceso de aire & $20 \%$ \\
Temperatura ambiental & $30{ }^{\circ} \mathrm{C}$ \\
Temperatura agua de alimentación & $28{ }^{\circ} \mathrm{C}$ \\
Temperatura del combustible & $28{ }^{\circ} \mathrm{C}$ \\
Presión atmosférica & $97,53 \mathrm{kPa}$ \\
$\mathrm{N}^{\circ}$ pasos en la caldera & 2 \\
Longitud del hogar total & $1,00 \mathrm{~m}$ \\
Longitud efectiva del hogar & $0,75 \mathrm{~m}$ \\
Diámetro interior del hogar & $0,25 \mathrm{~m}$ \\
Diámetro exterior del hogar & $0,30 \mathrm{~m}$ \\
Longitud del haz de tubos & $0,85 \mathrm{~m}$ \\
Longitud efectiva del haz de tubos & $0,75 \mathrm{~m}$ \\
Diámetro interior del tubo & $0,057 \mathrm{~m}$ \\
Diámetro exterior del tubos & $0,067 \mathrm{~m}$ \\
$\mathrm{~N}^{\circ}$ de tubos en el haz & 21 \\
Diámetro exterior de la coraza & $0,83 \mathrm{~m}$ \\
Espesor de la coraza & $0,002 \mathrm{~m}$ \\
Tipo de aislante & Lana vidrio \\
Espesor del aislante & $0,055 \mathrm{~m}$ \\
\hline \hline
\end{tabular}

Se describe el código utilizado en el programa EES para solucionar las ecuaciones del modelo matemático, y así compararlas con los resultados de la simulación [16].

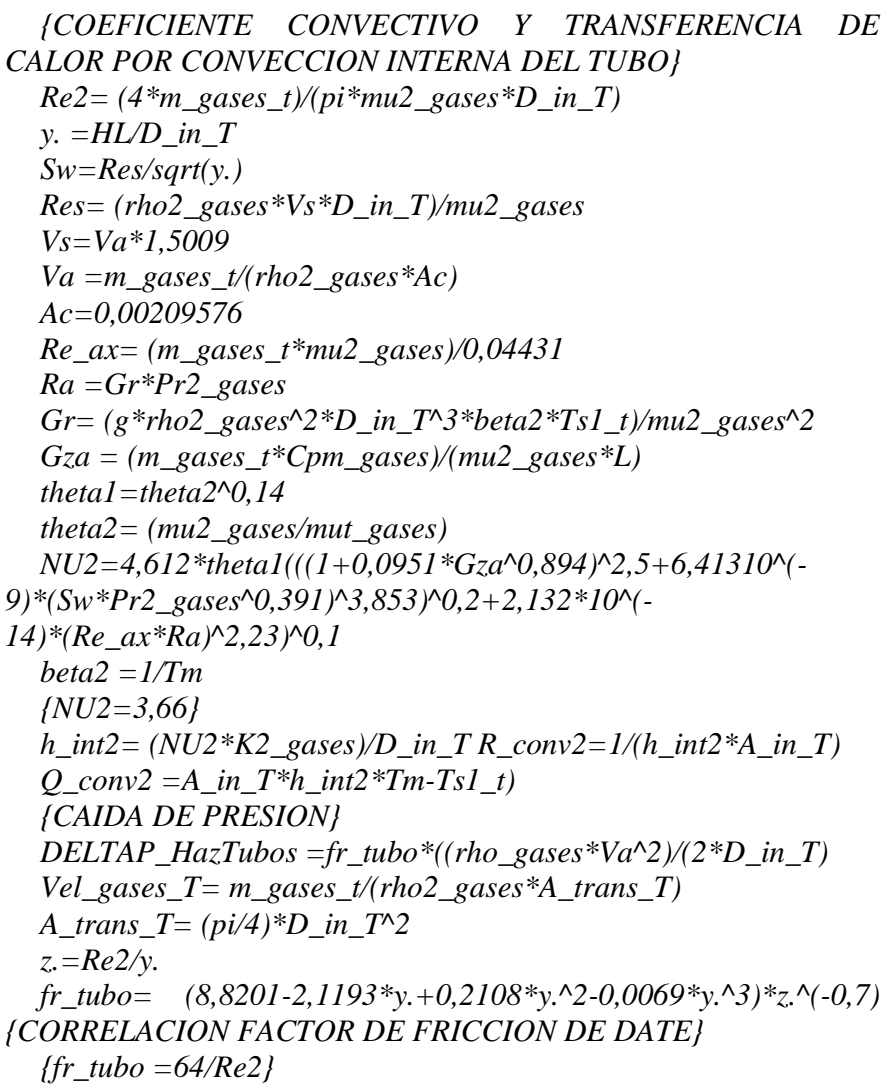


Los siguientes resultados se obtuvieron del funcionamiento de una caldera pirotubular, cuyo combustible es Diésel o ACPM $\left(\mathrm{C}_{12} \mathrm{H}_{26}\right)$, presión de operación de $310 \mathrm{kPa}(44,96 \mathrm{Psi})$ y $22 \%$ de exceso de aire. En la Tabla IV, se observan las diferentes características del funcionamiento de un componente del haz de tubos, bajo los efectos del turbulador, y se compara con el desempeño del mismo bajo condiciones normales.

TABLA IV

COMPARACIÓN DEL FUNCIONAMIENTO DE LA CALDERA CON Y SIN TURBULADOR

\begin{tabular}{ccc}
\hline \hline $\begin{array}{c}\text { Poperacion }=310 \text { kPa } \\
\text { Reynolds }=\mathbf{4 0 9 , 1}\end{array}$ & $\begin{array}{c}\text { Desempeño } \\
\text { sin turbulador }\end{array}$ & $\begin{array}{c}\text { Desempeño } \\
\text { con } \\
\text { turbulador }\end{array}$ \\
\hline Factor de Fricción $[\mathrm{fr}]$ & 0,1564 & 0,1163 \\
Numero Nusselt $[\mathrm{Nu}]$ & 3,66 & 5,325 \\
$\quad \begin{array}{c}\text { Coeficiente de transferencia de } \\
\text { calor convectivo }\left[\mathrm{W} / \mathrm{m}^{2} \mathrm{~K}\right]\end{array}$ & 3,241 & 4,595 \\
$\quad$ Calor de convección $[\mathrm{W}]$ & 123,6 & 162,2 \\
Caída de presión $[\mathrm{kPa}]$ & 0,2077 & 0,2240 \\
\hline \hline
\end{tabular}

La simulación muestra los resultados del flujo de calor que se transfiere por la pared del volumen de control en la figura 6 , considerando el proceso de conducción de calor por un tubo de acero A515 grado 70 cuya conductividad térmica es 52,922 $\mathrm{W} / \mathrm{m}-\mathrm{K}$. [8]

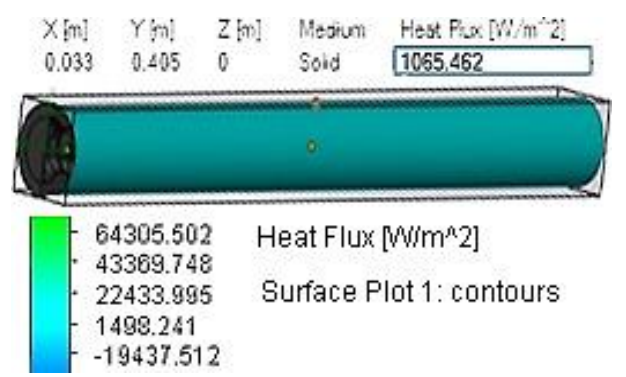

Fig. 6. Transferencia de calor al exterior del tubo. Fuente. Elaboración propia.

La Fig. 7, muestra la distribución de temperatura de los gases de combustión, según la trayectoria que adquieran estos mismos. A medida que entran al turbulador, tienden a calentarse debido al aumento de presión en las partículas por el cambio de área del objeto

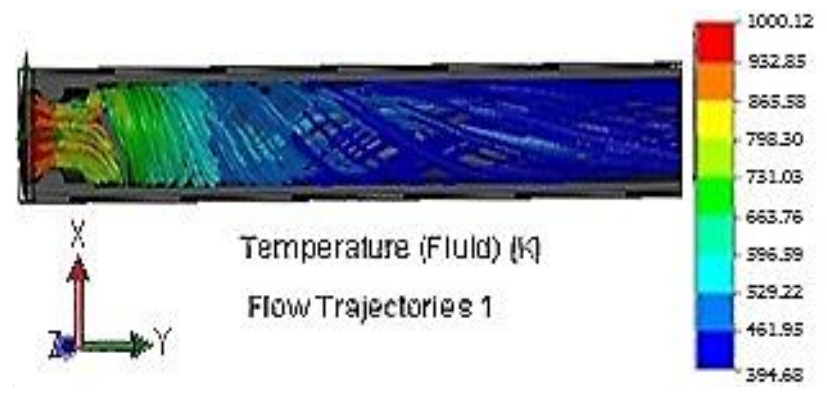

Fig. 7. Distribución de temperaturas bajo efectos del turbulador. Fuente. Elaboración propia.
A medida que los productos de combustión atraviesan el tubo, estos pierden energía. En la Fig. 7 se evidencia la disminución de la temperatura, y en la Fig. 8 la reducción de la componente de velocidad. Esto ocurre porque la energía de los gases es entregada a la siguiente etapa del proceso de generación de vapor, el calentamiento del fluido de trabajo y posteriormente el cambio de fase bajo las condiciones deseadas.

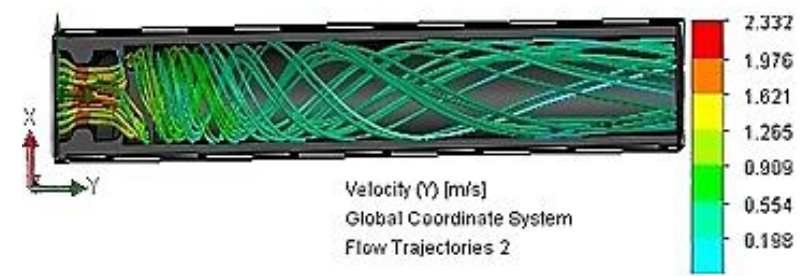

Fig. 8. Velocidad de los gases de combustión en el turbulador. Fuente. Elaboración propia.

La Fig. 9, muestra a detalle la trayectoria que adquieren los gases de combustión al pasar por el turbulador. Los gases son mezclados de una forma más uniforme. Asimismo, como tienden a estar en contacto continuo con el tubo que los rodea se genera un componente extra de calor por fricción. Los gases no viajan por el centro del tubo, por lo tanto, no se desperdicia la energía por efectos del perfil de velocidad de flujo laminar.

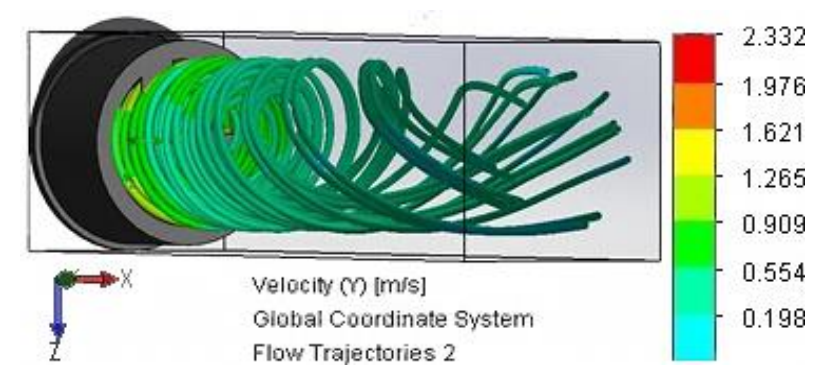

Fig. 9. Trayectoria de los gases al cruzar el turbulador. Fuente. Elaboración propia.

\section{CONCLUSIONES}

Se registra un incremento de $38,6 \mathrm{~W}$ de la transferencia de calor en un tubo del haz al instalar el turbulador, esto quiere decir que los turbuladores aumentan cerca del $31,23 \%$ el desempeño de calentamiento de una caldera. Sin embargo, la caída de presión aumenta $0,0163 \mathrm{kPa}$, ocasionado por el cambio de velocidad al pasar por el dispositivo.

La simulación muestra un flujo de calor de $1065,462 \mathrm{~W} / \mathrm{m}^{2}$. Debido a que el área transversal del tubo es de $0,1583 \mathrm{~m}^{2}$ el calor neto transferido es de $168,66 \mathrm{~W}$. Por medio de las ecuaciones presentadas en el desarrollo matemático del artículo, se obtiene una transferencia de calor por convección de $162,2 \mathrm{~W}$ a una presión de operación de $310 \mathrm{kPa}$. Esto quiere decir que los parámetros seleccionados en la simulación son validados por medio del modelo matemático ya que la diferencia en los resultados es mínima.

La temperatura a la que salen los productos de combustión es de $394,68 \mathrm{~K}$, aceptable por la norma técnica para emisiones de gases. 
Al momento que los gases de combustión entran al turbulador, se produce un aumento de la velocidad de los mismos, logrando un máximo de 2,332 m/s. Sin embargo, al momento de expandirse esta disminuye aproximadamente a $0,2 \mathrm{~m} / \mathrm{s}$.

La presencia de turbuladores mejora la transferencia de calor por la fricción de los gases con las paredes de los tubos. De lo contrario el perfil de velocidad seria laminar, lo que implicaría el aumento de la superficie de contacto entre el fluido con el recipiente que lo contiene.

Los turbuladores son dispositivos que permiten mejorar los mecanismos de transferencia de calor al interior de las calderas de tubos de fuego, del mismo modo que se aumenta la eficiencia de la máquina.

\section{REFERENCIAS}

[1] J. Marín y S. Guillen, "Diseño y calculo de intercambiadores de calor monofásicos", $1^{\circ}$ Edición, Madrid, España: Paraninfo Editorial, 2013.

[2] J. Hartnett and T. Irvine, "Advances in heat transfer", Chicago, Illinois, U.S.A.: Academic Press, Elsevier Science, Vol 36, 2002.

[3] F. Incropera, D. de Witt, T. Bergman \& A. Lavine, "Fundamentals of Heat and Mass Transfer", New York, U.S.A.: $7^{\circ}$ Edition. John Wiley \& Sons Ed., 2011.

[4] B. Munson, D. Young y T. Okiishi, "Fundamentos de Mecánica de Fluidos", $4^{\circ}$ Edición. Nueva York, U.S.A.: John Wiley \& Sons Ed., 2002.

[5] S. Kakac, H. Liu \& A. Pramuanjaroenkij, "Heat exchangers, selection, rating and thermal design", New York, U.S.A., $3^{\circ}$ Edition. CRC Press, 2012.

[6] J. Castro, "Operación y Mantenimiento de Calderas", $1^{\circ}$ Edición, Bogotá, Colombia: Universidad Nacional De Colombia, 2002.

[7] W. Rohsenow, J. Hartnett and Y. Cho, "Handbook of heat transfer”, $3^{\circ}$ Edición, New York, U.S.A.: McGraw-Hill, 1998.

[8] R. Manglik and A. Bergles, "Swirl Flow Heat Transfer and Pressure Drop with Twisted-Tape Inserts", Advances in Heat Transfer, Volume 36, pp. 183-266, 2003, DOI: 10.1016/S00652717(02)80007-7.

[9] Y. Cengel \& A. Ghajar, "Transferencia de calor y masa". Nueva York, U.S.A., $4^{\circ}$ Edición, Ed. McGraw-Hill, 2011.

[10] A. Date and J. Singham, "Numerical prediction of friction and heat transfer characteristics of fully developed laminar flow in tubes containing twisted tapes", New York, U.S.A.: ASME. 1972.

[11] B. Arévalo, E. Bayona, y P. Rincón. "Metodologia para documentacion 3D utilizando fotogrametria digital." Revista Tecnura, Vol 19, pp. 113-120, 2015. DOI: 10.14483/22487638.10377.

[12] G. Defranco, L. Fuertes, S. Gavino, L. Lopresti, M. Lara \& S. Barba. "Nuevas técnicas de relevamiento: el photo scanning". Segundas Jornadas de Investigación y Transferencia. Issue Mayo, pp. 672-677. 2013. http://sedici.unlp.edu.ar/handle/10915/38241.

[13] M. Pueo y J. Sierra-Pérez, "La introducción del modelado paramétrico 3D como herramienta multidisciplinar para los futuros desafíos del ejército de tierra", Centro Universitario de la Defensa UNIZAR, Zaragoza, España. 2014.

[14] Dassault Systemes, "Guia de usuario Solidworks Flow Simulation", 2016.

[15] J. Matsson, "An introduction to SolidWorks Flow Simulation", Edition 013, U.S.A:, SDC Publications, 2013.

[16] S. A. Klein, "Handbook Engineering Equation Solver", EES. FChart Software, 2016.

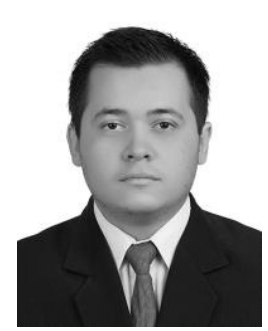

Cristian Ricardo Rubio Ramirez. Nació en Cúcuta, Colombia. Es ingeniero mecánico graduado en 2016 de la Universidad Francisco de Paula Santander, Cúcuta, Colombia. Miembro del grupo de investigación FLUTER. Actualmente es estudiante de maestría en ingeniería mecánica en la Universidad Federal de Rio Grande del Sur, Porto Alegre Brasil. Sus intereses investigativos incluyen, procesos y equipamientos térmicos, procesos de fabricación y modelado y simulación numérica.

ORCID: http://orcid.org/0000-0002-7709-8136

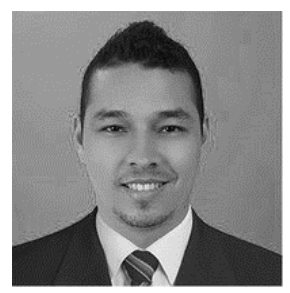

Guillermo Alfredo Martheyn Lizarazo. Nació en Cúcuta, Colombia. Es ingeniero mecánico graduado en 2016 de la Universidad Francisco de Paula Santander, Cúcuta, Colombia. Miembro del grupo de investigación FLUTER. Actualmente es estudiante de maestría en educación en la Universidad Internacional de La Rioja, España. Sus intereses investigativos incluyen, procesos térmicos, análisis, modelado y simulación numérica de fenómenos térmicos de transporte.

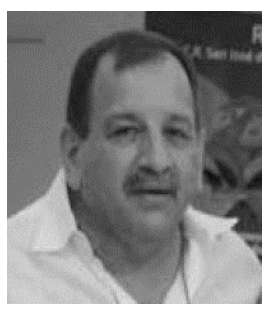

Luis Emilio Vera Duarte. Nació en Cúcuta, Colombia. Es ingeniero mecánico graduado en 1986 de la Universidad Francisco de Paula Santander, Cúcuta, Colombia. Posee una maestría de la Universidad Nacional y Experimental del Táchira, Mención Termofluidos, y una especialización de la Universidad Francisco de Paula Santander en Gestión Ambiental con Énfasis Saneamiento. Actualmente es docente investigador de la Universidad Francisco de Paula Santander y miembro del grupo de investigación FLUTER. Sus intereses investigativos incluyen, termodinámica aplicada, combustión y fenómenos de transporte.

ORCID: https://orcid.org/0000-0001-8756-7779 